\title{
VCAM-1 is critical in atherosclerosis
}

\author{
Klaus Ley and Yuqing Huo \\ Department of Biomedical Engineering, University of Virginia Health Science Center, \\ Charlottesville, Virginia, USA
}

Address correspondence to: Klaus Ley, Department of Biomedical Engineering, University of Virginia Health Science Center, Box 800759, Charlottesville, Virginia 22908, USA. Phone: (804) 924-1722; Fax: (804) 982-3870; E-mail: klausley@virginia.edu.

\begin{abstract}
VCAM-1 is an immunoglobulin-like adhesion molecule expressed on activated endothelial cells (1). VCAM-1 binds to $\alpha_{4} \beta_{1}$ integrin, which is constitutively expressed on lymphocytes, monocytes, and eosinophils. Interestingly, VCAM-1 can mediate both rolling-type adhesion and firm adhesion, depending on the avidity status of $\alpha_{4} \beta_{1}$ integrin (2). Although it is structurally similar to ICAM- 1 and other endothelial adhesion molecules, VCAM-1's pattern of regulation is unique. VCAM-1 is not expressed under baseline conditions but is rapidly induced by proatherosclerotic conditions in rabbits (3), mice (4), and humans (5), including in early lesions.
\end{abstract} Initially, it was unclear whether VCAM-1 was simply a marker for atherogenesis or whether it acts in this disease pathway. Studies with cytokine-activated cultured endothelial cells and reconstitution assays with purified recombinant VCAM-1 protein (2) suggested that VCAM-1 could mediate robust adhesion of $\alpha_{4} \beta_{1^{-}}$ expressing cells, even under shear flow. However, it remained unclear whether this adhesion could occur in vivo in large arteries of atherosclerosis-prone animals or patients.

Indirect evidence supporting a pathogenic role for VCAM-1 came from in vivo assays showing that recruitment of peritoneal macrophages into atherosclerotic lesion of Apoe $e^{-/-}$mice can be inhibited by an $\alpha_{4}$ blocking antibody (6). This was further supported by evidence that chronic application of a small peptide that inhibits ligand binding by $\alpha_{4} \beta_{1}$ delays the development of atherosclerosis in mice fed an atherogenic diet (7). However, interpretation of this study was complicated by the fact that activated endothelial cells can express a second ligand for $\alpha_{4} \beta_{1}$ integrin, alternatively spliced fibronectin containing the CS- 1 peptide. $\alpha_{4} \beta_{1}$-mediated adhesion to fibronectin is blocked by the same peptide, and some cultured endothelial cells express only fibronectin but not VCAM-1 (8). Other studies, using perfused carotid arteries harvested from Apoe ${ }^{-/-}$mice, showed that interactions between $\alpha_{4} \beta_{1}$ and VCAM- 1 can slow down rolling monocytes (9) and that VCAM-1 promotes monocyte adhesion and accumulation on the vessel wall at sites that are prone to developing atherosclerotic lesions (10). Although highly suggestive, these studies stopped short of providing conclusive evidence for a functional role for VCAM-1. This became possible only in VCAM-1 knockout mice.

Because of the suspected importance of VCAM-1 in atherosclerosis and other inflammatory diseases, knockout strategies were devised early (11). However, no or excruciatingly few pups homozygous for the null allele were born, apparently because VCAM1 's interactions with $\alpha_{4} \beta_{1}$ are required for successful chorio-allantoic fusion (11). Although VCAM-1 knockout mice were unavailable, other adhesion molecule-deficient mice have been tested successfully in various models of atherosclerosis. Working with a C57BL/6 mouse model fed a high-cholesterol diet containing cholate, Nageh et al. (12) showed that aortic lesion size is significantly reduced in ICAM-1 or P-selectin null mice or in mice with a hypomorphic mutation in the gene encoding the common CD18 subunit of $\beta_{2}$ integrins. In the more realistic apoE knockout model of atherosclerosis, in which mice have cholesterol levels between 500 and $2,000 \mathrm{mg} / \mathrm{dl}$, depending on the diet offered, animals lacking P-selectin or ICAM-1 show significantly smaller aortic lesions (13, 14). In a milder model of atherosclerosis, the LDL receptor knockout mouse, absence of P-selectin showed only a modest benefit in male mice and no benefit in female mice (15).
The study in this issue of the JCI shows, for the first time, that VCAM-1 is critical for the development of atherosclerotic lesions in this LDL receptor knockout mouse model (16). This is important because the LDL receptor knockout mouse is a good model of human atherosclerosis and develops significant lesions when fed a proatherogenic diet. To increase the number of surviving $V C A M-1^{-/-}$pups, Cybulsky and coworkers removed only one of the two ligand binding sites of VCAM-1, resulting in the D4D mutation. This mutant lacks domain 4 of VCAM-1 and its expression is much lower than that of wild-type VCAM-1, but it provides enough function (through domain 1) to allow 6-24\% of the homozygous mutant mice to survive to birth and beyond, depending on the strain. The degree of protection from atherosclerosis in the VCAM$1^{D 4 D / D 4 D} L D L R^{-/-}$mice is stronger than that of other mutants tested in the LDL receptor knockout model, suggesting that VCAM-1 is indeed a very important gene product directly involved in the formation of atherosclerotic lesions. In the same model, these authors find that a hypomorphic mutation of ICAM-1 has no effect on lesion size, either alone or in combination with the $V C A M^{4 D / 4 D}$ mutation.

This landmark study is not without limitations. First, the mice were not fully backcrossed into a homogeneous mouse strain. Only $6 \%$ of $V C A M-1^{4 D / 4 D}$ pups survive in the C57BL/6 strain, too few to conduct meaningful studies. Conditional VCAM-1 knockout mice, which could offer another way to circumvent the problem of developmental lethality, have been described $(17,18)$ but have not yet been tested in atherosclerosis models. It is possible that inducible and/or tissue-specific VCAM1 knockouts may provide informative data when crossed with the LDL receptor knockout mutants. Second, 
although the LDL receptor knockout is a good model of atherosclerosis, it is not perfect. Much of the elevated cholesterol is in VLDL in these mice, rather than in LDL. In humans, LDL (and not VLDL) cholesterol is considered one of the most important risk factors for atherosclerosis. Better mouse models exist, including transgenic mice expressing human apoB on an LDL receptor knockout background (19).

Beyond the formation of atherosclerotic lesions addressed in the present study, VCAM-1 may also be important in neointima formation after arterial injury, an area of research important for restenosis. In the wire-induced injury model, VCAM-1 expression is highly induced in the neointimal smooth muscle cells (20), and a VCAM-1 antibody protects from neointima formation (21). These findings suggest that VCAM-1 participates in neointima formation perhaps because it facilitates monocyte infiltration into injured arteries or because it directly enhances smooth muscle cell proliferation. These possibilities can now be tested using VCAM$1^{4 D / 4 D}$ mice. The availability of this new mutant VCAM-1 allele also open new perspectives for studies in other VCAM1-dependent disease models, including experimental autoimmune encephalitis, inflammatory bowel disease, and various arthritis models. The fact that the $V C A M-1^{4 D}$ allele is hypomorphic rather than a null mutation can be viewed as an advantage, because it may better mimic the incomplete blockade of VCAM-1 function that might result from therapeutic intervention. This mouse promises to be an invaluable tool in many areas of research into vascular and inflammatory diseases.

1. Osborn, L., et al. 1989. Direct expression cloning of vascular cell adhesion molecule 1 , a cytokineinduced endothelial protein that binds to lymphocytes. Cell. 59:1203-1211.

2. Chen, C., et al. 1999. High affinity very late antigen- 4 subsets expressed on $T$ cells are mandatory for spontaneous adhesion strengthening but not for rolling on VCAM-1 in shear flow. J. Immunol. 162:1084-1095.

3. Cybulsky, M.I., and Gimbrone, M.A., Jr. 1991 Endothelial expression of a mononuclear leukocyte adhesion molecule during atherogenesis. Science. 251:788-791.

4. Nakashima, Y., Raines, E.W., Plump, A.S., Breslow, J.L., and Ross, R. 1998. Upregulation of VCAM-1 and ICAM-1 at atherosclerosis-prone sites on the endothelium in the apoe-deficien mouse. Arterioscler. Thromb. Vasc. Biol. 18:842-851.

5. O'Brien, K.D., et al. 1993. Vascular cell adhesion molecule- 1 is expressed in human coronary atherosclerotic plaques. Implications for the mode of progression of advanced coronary atherosclerosis. J. Clin. Invest. 92:945-951.

6. Patel, S.S., Thiagarajan, R., Willerson, J.T., and Yeh, E.T.H. 1998. Inhibition of $\alpha_{4}$ integrin and ICAM-1 markedly attenuate macrophage homing to atherosclerotic plaques in apoE-deficient mice. Circulation. 97:75-81.

7. Shih, P.T., et al. 1999. Blocking very late antigen4 integrin decreases leukocyte entry and fatty streak formation in mice fed an atherogenic diet. Circ. Res. 84:345-351.

8. Shih, P.T., et al. 1999. Minimally modified lowdensity lipoprotein induces monocyte adhesion to endothelial connecting segment- 1 by activating beta 1 integrin. J. Clin. Invest. 103:613-625

9. Ramos, C.L., et al. 1999. Direct demonstration of P-selectin- and VCAM-1-dependent mononuclear cell rolling in early atherosclerotic lesions of apolipoprotein E-deficient mice. Circ. Res. 84:1237-1244
10. Huo, Y., Hafezi-Moghadam, A., and Ley, K. 2000 Role of vascular cell adhesion molecule- 1 and fibronectin connecting segment- 1 in monocyte rolling and adhesion on early atherosclerotic lesions. Circ. Res. 87:153-159.

11. Gurtner, G.C., et al. 1995. Targeted disruption of the murine VCAM-1 gene: essential role of VCAM-1 in chorioallantoic fusion and placentation. Genes Dev. 9:1-14.

12. Nageh, M.F., et al. 1997. Deficiency of inflammatory cell adhesion molecules protects against ath erosclerosis in mice. Arterioscler. Thromb. Vasc. Biol. 17:1517-1520.

13. Collins, R.G., et al. 2000. P-Selectin or intercellular adhesion molecule (ICAM)-1 deficiency substantially protects against atherosclerosis in apolipoprotein E-deficient mice. J. Exp. Med. 191:189-194.

14. Dong, Z.M., Brown, A.A., and Wagner, D.D. 2000. Prominent role of P-selectin in the development of advanced atherosclerosis in ApoE-deficient mice. Circulation. 101:2290-2295.

15. Johnson, R.C., et al. 1997. Absence of P-selectin delays fatty streak formation in mice. J. Clin. Invest. 99:1037-1043.

16. Cybulsky, M.I., et al. 2001. A major role for VCAM-1, but not ICAM-1, in early atherosclerosis. J. Clin. Invest. 107:xxxx-yyyy.

17. Koni, P.A., et al. 2001. Conditional vascular cel adhesion molecule 1 deletion in mice. impaired lymphocyte migration to bone marrow. J. Exp. Med. 193:741-754.

18. Leuker, C.E., Labow, M., Muller, W., and Wagner, N. 2001. Neonatally induced inactivation of the vascular cell adhesion molecule 1 gene impairs $B$ cell localization and $\mathrm{T}$ cell-dependent humoral immune response. J. Exp. Med. 193:755-768.

19. Powell-Braxton, L., et al. 1998. A mouse model of human familial hypercholesterolemia: markedly elevated low density lipoprotein cholesterol levels and severe atherosclerosis on a low-fat chow diet. Nat. Med. 4:934-938.

20. Manka, D.R., et al. 1999. Arterial injury increases expression of inflammatory adhesion molecules in the carotid arteries of apolipoprotein-E-deficient mice. J. Vasc. Res. 36:372-378.

21. Oguchi, S., et al. 2000. Monoclonal antibody against vascular cell adhesion molecule- 1 inhibits neointimal formation after periadventitial carotid artery injury in genetically hypercholesterolemic mice. Arterioscler. Thromb. Vasc. Biol. 20:1729-1736. 